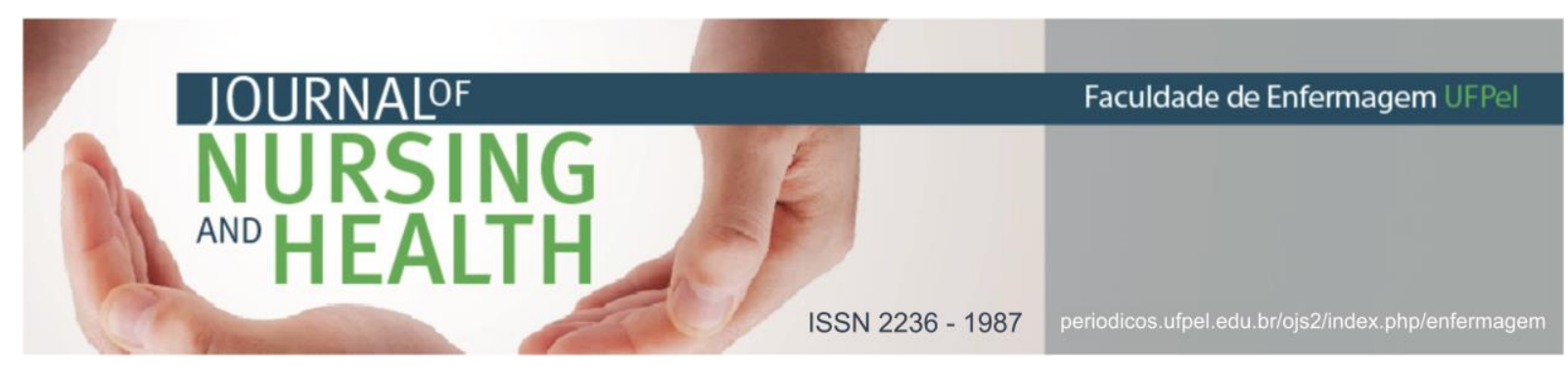

ARTIGO DE REVISÃO

\title{
Implicações da polimedicação em idosos portadores de osteoporose
}

\author{
Implications of polypharmacy in elderly people with osteoporosis \\ Implicaciones de la polifarmacia en pacientes ancianos con osteoporosis
}

Edivaldo CREMER ${ }^{1}$, Maria José Quina GALDINO², Julia Trevisan MARTINS ${ }^{3}$

\begin{abstract}
RESUMO
Objetivo: analisar a produção científica sobre as possíveis implicações da polimedicação em idosos em tratamento de osteoporose. Métodos: revisão integrativa de literatura realizada por busca no Portal de Periódicos da CAPES abrangendo artigos publicados nos últimos cinco anos. Foram obtidos 814 artigos e após a seleção foram incluídos nove estudos. Resultados: a polimedicação entre idosos com osteoporose aumentou o risco de quedas com consequentes fraturas, sobretudo devido ao uso de medicamentos ansiolíticos, hipnóticos, analgésicos opióides e com ação cardiovascular. Conclusão: a polimedicação em idosos em tratamento de osteoporose deve ser criteriosa, baseada no quadro clínico e realizada quando os benefícios superarem os malefícios.

Descritores: Osteoporose; Idoso; Polimedicação; Efeitos colaterais e reações adversas relacionados a medicamentos.
\end{abstract}

\begin{abstract}
Objective: to analyze the scientific production on the possible implications of polypharmacy in the elderly in the treatment of osteoporosis. Methods: integrative review of literature conducted by searching the Portal de Periódicos da CAPES covering articles published in the last five years. 814 articles were obtained and nine studies were included after the selection. Results: polypharmacy among elderly patients with osteoporosis increased the risk of falls with consequent fractures, mainly due to the use of anxiolytic drugs, hypnotics, opioid analgesics and cardiovascular action. Conclusion: polypharmacy in the elderly in the treatment of osteoporosis should be judicious, based on the clinical picture and performed when the benefits outweigh the harm.

Descriptors: Osteoporosis; Aged; Polypharmacy; Drug-related side effects and adverse reactions.
\end{abstract}

\section{RESUMEN}

Objetivo: analizar la literatura científica sobre las posibles implicaciones de la polifarmacia en los ancianos en el tratamiento de la osteoporosis. Métodos: revisión integradora de la literatura mirando el Portal de Periódicos da CAPES que cubre los artículos publicados en los últimos cinco años. Se obtuvieron 814 artículos y después de la selección se incluyeron nueve estudios. Resultados:

${ }^{1}$ Enfermeiro. Doutorando em Ciências da Saúde da Universidade Estadual de Maringá. Professor Assistente da Universidade Estadual do Norte do Paraná, Bandeirantes, Paraná, Brasil. E-mail: edivaldocremer@gmail.com

Enfermeira. Doutoranda em Enfermagem da Universidade Estadual de Maringá. Professor Assistente da Universidade Estadual do Norte do Paraná, Bandeirantes, Paraná, Brasil. E-mail: mariagaldino@uenp.edu.br ${ }^{3}$ Enfermeira. Doutora em Enfermagem. Professora Associada da Universidade Estadual de Londrina. Londrina, Paraná, Brasil. E-mail: jtmartins@uel.br 


\section{JOURNALOF

polifarmacia en los ancianos con osteoporosis aumenta el riesgo de caídas y fracturas resultantes, principalmente debido al consumo de ansiolíticos, hipnóticos, analgésicos opioides y la acción cardiovascular. Conclusión: la polifarmacia en personas de edad avanzada en el tratamiento de la osteoporosis deben tener cuidado, basado en clínica y lleva a cabo cuando los beneficios superan los daños.

Descriptores: Osteoporosis; Anciano; Polifarmacia; Efectos colaterales y reacciones adversas relacionados con medicamentos.

\section{INTRODUÇÃO}

0 uso de medicamentos por idosos teve um crescimento significativo nos últimos anos devido a maior prevalência de doenças crônicas não transmissíveis (DCNT) e as manifestações clínicas que acompanham 0 processo de envelhecimento. Assim, a polimedicação nessa população é comum e influenciada pelo poder da indústria farmacêutica, pelo marketing dos medicamentos e pela medicalização estar presente na formação da maioria dos profissionais da saúde. ${ }^{1-3}$

A polimedicação é definida como o uso simultâneo e de forma crônica de vários medicamentos (geralmente 5 ou mais) quer seja, por meio de prescrições ou pela automedicação. ${ }^{4}$ Dessa forma, o uso de um ou a associação medicamentos de deve ser foco da atenção dos profissionais de saúde, familiares e da própria pessoa.

Em pesquisa de base populacional denominada Estudo Saúde, Bem-estar e Envelhecimento (SABE) desenvolvida no município de São Paulo identificouse alta prevalência da polimedicação entre a população estudada e concluíram que o uso indiscriminado pode levar a graves consequências para este grupo etário. ${ }^{5}$

Destaca-se que os idosos podem fazer uso de cinco ou mais medicações desde que tenha acompanhamento médico regularmente, bem como que os benefícios ultrapassem os malefícios de sua utilização, porém é preocupante o uso de medicamentos potencialmente inapropriados. Estudos mostraram que idosos em uso desses medicamentos apresentaram maior risco de morbi-mortalidade e de apresentar uma reação adversa. ${ }^{6-7}$

A polimedicação, sobretudo entre os idosos com DCNT, tem sido uma preocupação para os governos em todo mundo, configurando-se como um problema de saúde pública. Assim sendo, é fundamental importância que os profissionais ao prescreverem o medicamento avaliem a sua adequação, não apenas em relação à escolha do mesmo, mas também quanto à dosagem a ser utilizada. ${ }^{8}$

Dentre as principais DCNT presentes na população, cita-se a osteoporose uma doença esqueléticasistêmica caracterizada pela diminuição da densidade mineral óssea, que tem como principal consequência a fratura óssea $e$ acomete cerca de $17 \%$ mulheres e $7 \%$ homens. ${ }^{9}$ Contudo, por ser uma doença sem manifestações clínicas evidentes ao portador, deve se considerar sua subnotificação e, desse modo, estimase que seja a mais prevalente doença crônica em todo o mundo, especialmente em mulheres acima de 65 anos. $^{10}$ 
Diante do exposto, percebe-se a relevância desta temática para a área da saúde, em sua perspectiva biopsicossocial, seja pelo aumento da população de idosos ou pela multipatologia, sobretudo as DCNT, que frequentemente são mais prevalentes no processo de envelhecimento. Dessa maneira, por meio da compreensão das implicações da polimedicação para os idosos portadores de osteoporose, será possível subsidiar ações de promoção de saúde e, por consequência, o bemestar na senescência.

Com base neste contexto, objetivou-se analisar a produção científica sobre as possíveis implicações da polimedicação em idosos em tratamento de osteoporose.

\section{MATERIAIS E MÉTODOS}

Esta investigação caracteriza-se como uma revisão integrativa de literatura. A coleta de dados foi desenvolvida entre os meses de agosto a outubro de 2015. Optou-se por utilizar este método por permitir a inclusão de estudos com diferentes abordagens metodológicas e por reunir resultados de um conjunto de pesquisas com temáticas similares. ${ }^{11}$

Para realizar esta revisão integrativa adotaram-se as seguintes etapas: definição da questão de pesquisa, busca na literatura, categorização dos estudos, avaliação dos estudos incluídos, interpretação dos resultados e apresentação da revisão. ${ }^{11}$ Assim, definiu-se a seguinte pergunta de pesquisa: Quais são as implicações da polimedicação em idosos portadores de osteoporose?
Optou-se por pesquisar artigos indexados no Portal de Periódicos da CAPES, que integra diversas bases de dados, no qual é possível acessar o texto completo das publicações disponíveis. Estabeleceu-se como critérios de inclusão das publicações: artigos disponíveis eletronicamente na íntegra, sobre o tema proposto; artigos publicados em português, espanhol e inglês, no período de julho de 2011 a junho de 2015, a fim de levantar o conhecimento produzido nos cinco últimos anos sobre a temática em questão. Excluíram-se deste estudo editoriais, cartas, artigos de opinião, comentários, ensaios, notas prévias, revisões integrativas e sistemáticas de literatura, bem como as publicações repetidas, teses, dissertações e manuais.

As buscas foram realizadas entre os meses de julho e agosto de 2015. Para obter especificidade utilizaram-se os Descritores em Ciências da Saúde (DeCS) em diferentes combinações: "osteoporose/ osteoporosis", "polimedicação/ polypharmacy" e os descritores não controlados "reação adversa/adverse reactions" e "interação medicamentosa/drug interactions" para obter especificidade na busca.

Ao total, foram encontrados 814 artigos por meio da combinação dos descritores. 0 cruzamento dos termos "osteoporose" and "polimedicação" recuperou 1 artigo; dos termos "osteoporose" and "reação adversa" recuperou 5 artigos; dos termos "osteoporose" and "interação medicamentosa" recuperou 1 artigo; dos termos "osteoporosis" and "polypharmacy" recuperou 691 artigos; dos termos "osteoporosis" and 


\section{NURSING \\ aNo HEALTH}

"adverse reactions" recuperou 54 artigos; dos termos "osteoporosis" and "drug interactions" recuperou 62 artigos. Após a leitura dos resumos, 678 artigos foram descartados por não atenderem um ou mais critérios de inclusão ou se enquadrarem no critério de exclusão. Além disso, 127 artigos estavam repetidos. Assim, a amostra final foi constituída por nove publicações, as quais foram lidas na integra.

Para extrair os dados dos estudos selecionados elaborou-se um instrumento de coleta de dados, composto pelos seguintes itens: autores, título, ano de publicação, periódico científico, tipo de estudo, país de origem e conclusão.

A análise e interpretação dos dados foram realizadas após ampla leitura dos artigos, em que se sumarizou e organizou as informações; na sequência procedeu-se a comparação e fundamentação dos principais resultados com 0 conhecimento teórico; e, por fim, sistematizou-se todas as informações de cada artigo revisado.
ISSN 2236 - 1987

\section{RESULTADOS}

Os artigos selecionados foram publicados no período de 2011 a 2015, em periódicos de circulação internacional das áreas de farmacologia, medicina e alguns específicos de envelhecimento e osteoporose. A maioria dos estudos foram realizados em países europeus, por equipes multiprofissionais compostas principalmente por médicos e farmacêuticos. 55,6\% dos estudos foram realizados com pacientes de seguimento ambulatorial, 33,3\% com bancos de dados públicos e 11,1\% em lar de idosos.

Com relação ao tipo de abordagem metodológica utilizada nos artigos estudados nesta pesquisa, verificou-se que um estudo era ensaio clínico randomizado $(11,1 \%)$, cinco estudos de coorte $(55,6 \%)$; dois estudos transversais (22,2\%); um estudo descritivo $(11,1 \%)$. No quadro 1 podese verificar os artigos selecionados para este estudo, apresentando os autores, ano de publicação, título, país de origem, periódico cientifico, delineamento e conclusão do estudo.

\begin{tabular}{|c|c|c|c|c|c|}
\hline Autores & Título & País & Periódico & Delineamento & Principais resultados \\
\hline Belfrage et al. ${ }^{12}$ & $\begin{array}{l}\text { Number of drugs } \\
\text { in the } \\
\text { medication list } \\
\text { as an indicator } \\
\text { of prescribing } \\
\text { quality: a } \\
\text { validation study } \\
\text { of polypharmacy } \\
\text { indicators in } \\
\text { older hip } \\
\text { fracture } \\
\text { patients }\end{array}$ & Suécia & $\begin{array}{l}\text { European } \\
\text { Journal of } \\
\text { Clinical } \\
\text { Pharmac }\end{array}$ & $\begin{array}{l}\text { Coorte } \\
\text { prospectivo }\end{array}$ & $\begin{array}{l}\text { Além da polimedicação, } \\
\text { este estudo indicou a } \\
\text { prescrição } \\
\text { medicamentos } \\
\text { potencialmente } \\
\text { inapropriados, sendo que } \\
\text { muitas delas têm reações } \\
\text { adversas que aumentam o } \\
\text { risco de queda }\end{array}$ \\
\hline $\begin{array}{l}\text { Bakken et al. }{ }^{13} \\
2014\end{array}$ & 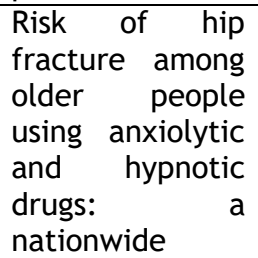 & $\begin{array}{l}\text { Norueg } \\
a\end{array}$ & $\begin{array}{l}\text { Europen } \\
\text { Journal of } \\
\text { Clinical } \\
\text { Pharmac }\end{array}$ & $\begin{array}{l}\text { Coorte } \\
\text { prospectivo }\end{array}$ & $\begin{array}{l}\text { Os idosos apresentaram um } \\
\text { risco aumentado de fratura } \\
\text { de quadril durante o uso de } \\
\text { ansiolíticos ou hipnóticos, } \\
\text { incluindo ansiolíticos, } \\
\text { benzodiazepínicos de ação } \\
\text { curta e hipnóticos que antes }\end{array}$ \\
\hline
\end{tabular}




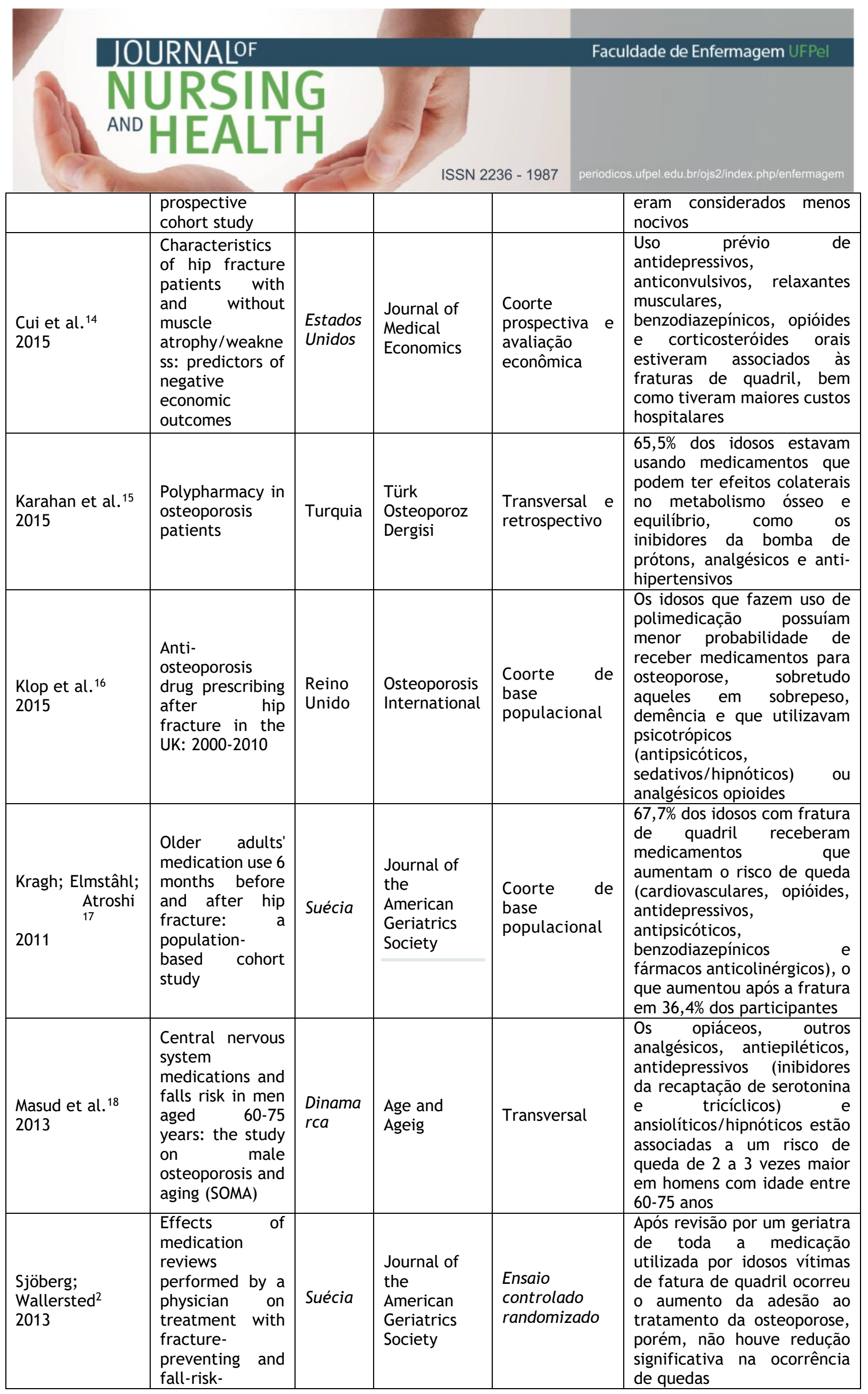




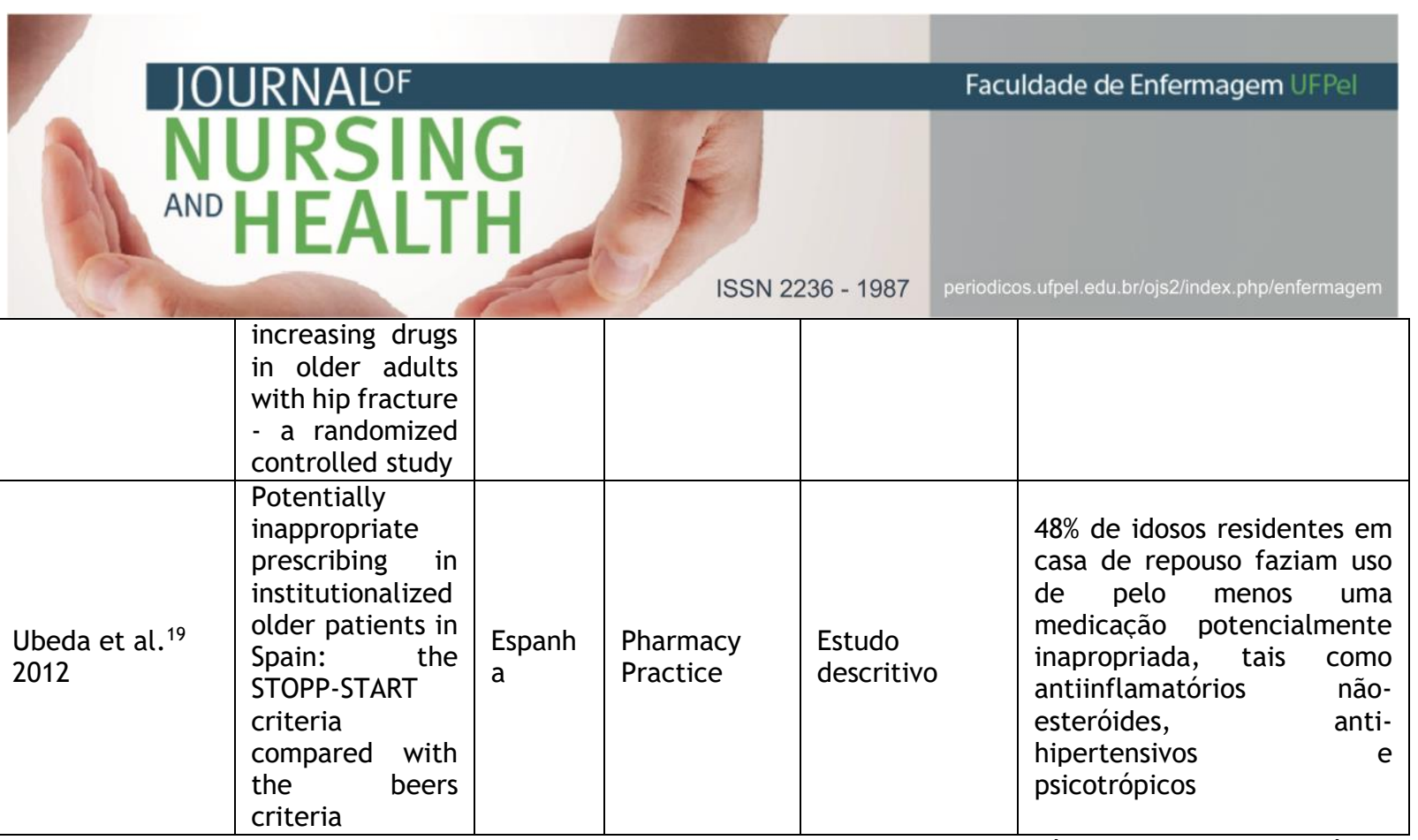

Quadro 1 - Distribuição dos estudos segundo autores, ano de publicação, título dos artigos, país de origem, periódico científico, delineamento e conclusão do estudo.

Fonte: Bandeirantes, 2015.

\section{DISCUSSÃO}

A quantidade de estudos incluídos para análise nesta pesquisa demonstrou que são escassas as investigações relacionadas à esse tema, especialmente as intervenções, que são capazes de gerar evidências científicas mais robustas. Ainda, verificou-se a valorização da temática em nível internacional, visto que esses estudos foram originados em países europeus e nos Estados Unidos, demonstrando-se, assim, a incipiência de pesquisas no âmbito nacional.

A polimedicação aumenta o risco de quedas com consequentes fraturas de quadril em cerca de $40 \%$, bem como o uso de três ou mais medicamentos psicotrópicos adiciona $8,6 \%$ à esta taxa. ${ }^{17}$

Em um estudo realizado na Turquia com 1000 idosos portadores de osteoporose identificou-se que $65 \%$ deles faziam uso de polimedicação e que $65.5 \%$ dos participantes tiveram problemas no metabolismo ósseo relacionado com o uso de algumas classes de medicações, sendo um fator determinante em $93,7 \%$ sobre o equilíbrio dos mesmos. ${ }^{15}$

Destaca-se ainda que os analgésicos, espasmolíticos analgésicos-opióides, antiparkinsonianos inflamatórios não-esteróides também já foram associados à um maior risco de queda, devido a hipotensão. ${ }^{16-17}$

Investigação realizada com 200 idosos suecos com fratura de quadril indicou o uso polimedicação em $72,5 \%$ da amostra, representados pelo uso de benzoazepínicos $(28,0 \%)$; diuréticos de alça sem sinais clínicos de insuficiência cardíaca (12,0\%); aspirina sem qualquer evento arterial oclusivo $(7,4 \%)$; neurolépticos - aqueles propensos a quedas $(7,4 \%)$; betabloqueadores $(6,0 \%)$; drogas vasodilatadoras conhecidas por causar hipotensão naqueles com hipotensão postural persistente $(6,0 \%)$; opiáceos de longo prazo em pessoas com quedas recorrentes $(6,0 \%)$; clopidogrel $(5.4 \%)$; varfarina $(4,7 \%)$; e estrogênios em pacientes com útero intacto $(4,0 \%)$. Além da polimedicação, este estudo indicou a prescrição de medicamentos sem a indicação clínica recomendada, 


\section{JOURNALOF \\ NURSING \\ AND HEALTH}

bem como sem levar em consideração que muitas delas têm reações adversas que aumentam o risco de queda. Também reflete que as doses ingeridas podem não ser as adequadas, considerando que esses idosos tomam suas medicações sozinhos e que, muitas vezes, seus estados cognitivos podem estar alterados em decorrência das reações adversas de algum medicamento. ${ }^{12}$

Uma investigação avaliou que haviam prescrições sem indicação clínica, e que, pela presença da polimedicação as drogas de tratamento para a osteoporose eram preteridas em relação aos outros fármacos para tratamento de outras doenças crônicas consideradas empiricamente como mais nocivas, tais como as doenças cardiovasculares. ${ }^{19}$ Os médicos podem avaliar que diante das outras doenças com maior potencial de mortalidade para o idoso - que repercute na quantidade de medicamentos por ele utilizados -, a osteoporose por seu perfil silencioso pode ser relegada, contudo deve se considerar o impacto de uma fratura na morbidade e no bem estar do paciente.

Em uma pesquisa realizada com 35.000 indivíduos americanos que sofreram fratura no quadril, e que tinham cobertura de um plano de saúde privado, identificou-se que o evento teve associação estatisticamente significante com indivíduos portadores concomitantes de osteoporose e atrofia muscular/fraqueza. Verificou-se uma elevada utilização de medicações simultâneas como os opioides, antidepressivos, corticoides orais, anti-inflamatórios não-esteroides, neurolépticos e relaxantes musculares
ISSN 2236 - 1987

há pelo menos um ano antes da fratura. ${ }^{14}$ Esse resultado ratifica a relação entre a medicalização, a osteoporose e as fraturas, o que deve ser um alerta para os profissionais, bem como para as pessoas e seus familiares no que diz respeito aos cuidados, orientações como a finalidade de prevenir agravos.

Os hipnóticos, antipsicóticos e antidepressivos foram associados maior risco de queda, como resultado de tonturas, hipotensão ortostática, sedação, ganho de peso, visão turva e confusão, reações adversas comuns desses medicamentos. ${ }^{20-22}$

Além dessas reações adversas, ocorre uma maior diminuição da densidade óssea nos indivíduos que fazem uso de neurolépticos e antidepressivos - inibidores seletivos da recaptação da serotonina; inibidores seletivos da recaptação da serotonina e da noradrenalina; inibidores da monoamina oxidase; e principalmente, os antidepressivos tricíclicos. ${ }^{21-23}$

Uma meta-análise identificou que - uso de benzodiazepínicos em pacientes com diagnóstico secundário de osteoporose, a taxa de fratura de quadril nas populações de 6 países: França, Alemanha, Itália, Espanha, Reino Unido e Estados Unidos, demonstrou um risco relativo de 1,4 (IC $95 \%$ ), que variou de $1,8 \%$ e $8,2 \%$ entre países, sendo atribuído ao maior ou menor consumo dessas drogas. ${ }^{24}$

Um ensaio clínico randomizado com 199 idosos com fratura de quadril atendidos em um hospital universitário sueco, no qual, realizou-se uma revisão em toda a medicação utilizada por eles com base em uma avaliação geral realizada por um geriatra. Esta 
intervenção aumentou a adesão ao tratamento da osteoporose, porém, não houve redução estatisticamente significativa na ocorrência de quedas, quando comparado ao grupo que não foi avaliado quanto à medicamentos potencialmente inapropriados. ${ }^{2}$

Apesar das limitações presentes neste estudo, relacionadas, sobremaneira, pelo baixo número de produções encontradas, em especial, a ausência de produções nacionais. Acredita-se que os resultados encontrados podem colaborar para discussões sobre o tema, bem como com o desenvolvimento de futuras pesquisas que abordem a temática polimedicação e sua relação com idosos portadores de osteoporose.

\section{CONSIDERAÇÕES FINAIS}

A maioria dos estudos relacionou a polimedicação à ocorrência de quedas, que devido a diminuição da densidade óssea provocada pela osteoporose aumenta as chances de fratura. Os medicamentos que predispõem ao risco de queda são aqueles utilizados para tratamento das doenças mais prevalentes na senescência, sobretudo os ansiolíticos, os hipnóticos, analgésicos opióides e com ação cardiovascular.

Esses resultados devem ser avaliados com parcimônia visto que muitas interações medicamentosas produzem positivos que aumentam a longevidade entre idosos com osteoporose. Assim, recomenda-se que a polimedicação seja realizada com cautela, baseada no quadro clínico e realizada quando os benefícios superarem os malefícios.

\section{REFERÊNCIAS}

1. Secoli SR. Polifarmácia: interações e reações adversas no uso de medicamentos por idosos. Rev bras enferm [Internet]. 2010[acesso em 2017 fev 10];63(1):136-40. Disponível em:

http://www.scielo.br/pdf/reben/v63 n1/v63n1a23.pdf

2. Sjöberg C, Wallerstedt SM. Effects of medication reviews performed by a physician on treatment with fracturepreventing and fall-risk-increasing drugs in older adults with hip fracture - a randomized controlled study. J am geriatr soc [Internet]. 2013[acesso em 2017 fev 10];61(9):1464-72. Disponível em:

http: / / onlinelibrary.wiley.com/doi/1 $0.1111 /$ jgs. 12412

3. Gautério DP, Santos SSC, Silva BT, Cruz VD, Zortea B, Alves I. Perfil sociodemográfico, diagnósticos e cuidados de enfermagem propostos para idosos institucionalizados que utilizam múltiplos medicamentos. J nurs health [Internet]. 2013[acesso em 2016 jan 10];3(2):182-94. Disponível em:

https: / /periodicos.ufpel.edu.br/ojs2/ index.php/enfermagem/article/view/ 3713

4. Patel RB. Polypharmacy and the elderly. J infus nurs [Internet]. 2003[acesso em 2017 fev 10];26(3):166-9. Disponível em: http://journals.lww.com/journalof infusionnursing/pages/articleviewer.a spx?year $=2003 \&$ issue $=05000 \&$ article $=0$ 0008

5. Carvalho MF, Romano-Lieber NS, Bergsten-Mendes G, Secoli SR, Ribeiro $E$, Lebrão $M L$, et al. Polypharmacy among the elderly in the city of São 


\section{JOURNALOF \\ NURSING \\ AND}

ISSN 2236 - 1987

Paulo, Brazil-SABE Study. Rev bras epidemiol [Internet]. 2012[acesso em 2017 fev 10];15:817-27. Disponível em: http://www.scielo.br/pdf/rbepid/v15 n4/13.pdf

6. Sichieri K, Rodrigues ARB, Takahashi JA, Secoli SR, Nobre MRC, Martinez MA, et al. Mortality associated with of the use of inappropriate drugs according Beers criteria: a systematic review. Adv pharm pharmacy [Internet]. 2013[acesso em 2017 fev 10]; 1:74-84. Disponível em: http: / /www.hrpub.org/download/201 310/app.2013.010205.pdf

7. Passarelli MCG, Jacob-Filho W, Figueras $A$. Adverse drug reactions in an elderly hospitalised population. Drugs aging [Internet]. 2005[acesso em 2017 fev 10]; 22:767-77. Disponível em:

http: / / link.springer.com/article/10.2 165/00002512-200522090-00005

8. Prybys KM, Melville K, Hanna J, Gee A, Chyka P. Polypharmacy in the elderly: clinical challenges in emergency practice: Part I: overview, etiology, and drug interactions. Emerg med rep [Internet]. 2002[acesso em 2017 fev 10];23:145-53. Disponível em: https: / /www.ahcmedia.com/articles/ 119992-polypharmacy-in-the-elderlyclinical-challenges-in-emergencypractice-part-i

9. Madureira MM, Bonfá E, Takayama L, Pereira RM. A 12-month randomized controlled trial of balance training in elderly women with osteoporosis: improvement of quality of life. Maturitas [Internet]. 2010[acesso em 2017 fev 10];66(2):206-11. Disponível em:

http: / /www.maturitas.org/article/S0 378-5122(10)00118-0
10. Fontes TMP, Araújo LFB, Soares PRG. Osteoporose no climatério I: epidemiologia, definição, rastreio e diagnóstico. Femina [Internet]. 2012[acesso em 2017 fev 10];40(2):109-16. Disponível em: http: / / www.febrasgo.org.br/site/ wpcontent/uploads/2013/05/Feminav40n2_109-116.pdf

11. Mendes KDS, Silveira RCCP, Galvão CM. Revisão integrativa: método de pesquisa para a incorporação de evidências na saúde e na enfermagem. Texto contexto enferm [Internet]. 2008[acesso em 2017 fev 10];17(4):758-64. Disponível em: http://www.scielo.br/pdf/tce/v17n4/ 18.pdf

12. Belfrage B, Koldestam A, Sjöberg C, Wallerstedt SM. Number of drugs in the medication list as an indicator of prescribing quality: a validation study of polypharmacy indicators in older hip fracture patients. Eur j clin pharmacol [Internet]. 2015[acesso em $2017 \mathrm{fev}$ 10];71:363-8. Disponível em: https://www.ncbi.nlm.nih.gov/pmc/a rticles/PMC4333233/

13. Bakken MS, Engeland A, Engesæeter LB, Ranhoff $A H$, Hunskaar S, Ruths S. Risk of hip fracture among older people using anxiolytic and hypnotic drugs: a nationwide prospective cohort study. Eur $j$ clin pharmacol [Internet]. 2014[acesso em 2017 fev 10];70:1972$9 . \quad$ Disponível em: https: / /www.ncbi.nlm.nih.gov/pmc/a rticles/PMC4053597/

14. Cui Z, Schoenfeld MJ, Bush EN, Chen Y, Burge R. Characteristics of hip fracture patients with and without muscle atrophy/weakness: predictors of negative economic outcomes. J med econ [Internet]. 2015[acesso em 2017 fev 10];18(1)1-11. Disponível em: 


\section{NURSING \\ AND}

ISSN 2236 - 1987

Pharm pract (Granada) [Internet]. 2012[acesso em 2017 fev 10];10(2):83$91 . \quad$ Disponível em: https: //www.ncbi.nlm.nih.gov/pmc/a rticles/PMC3780483/

20. Woolcott JC, Richardson KJ, Wiens MO, Patel B, Marin J, Khan KM, et al. Meta-analysis of the impact of 9 medication classes on falls in elderly person. Arch intern med [Internet]. 2009[acesso em 2017 fev 10];169(21):1952-60. Disponível em: https://www.ncbi.nlm.nih.gov/pubm edhealth/PMH0027943/

21. Rizzoli R, Cooper C, Reginster JY, Abrahamsen B, Adachi JD, Brandi ML, et al. Antidepressant medications and osteoporosis. Bone [Internet]. 2012[acesso em 2017 fev 10];51(3):606-13. Disponível em: http://www.thebonejournal.com/arti cle/S8756-3282(12)00915-5

22. Laguna-Parras JM, Arrabal-Orpez MJ, Zafra-López F, García-Fernández FP, Carrascosa-Corral RR, CarrascosaGarcía MI. Incidencia de caídas en un hospital de nivel 1: factores relacionados. Gerokomos [Internet]. 2011[acesso em 2017 fev 10];22(4):167-73. Disponível em: http://scielo.isciii.es/pdf/geroko/v22 n4/comunicacion3.pdf

23. Brown MJ, Mezuk B. Brains, bones, and aging: psychotropic medications and bone health among older adults. Curr osteoporos rep [Internet]. 2012[acesso em 2017 fev 10];10(4):303-11. Disponível em: https://www.ncbi.nlm.nih.gov/pmc/a rticles/PMC3508080/

24. Khong TP, Vries F, Goldenberg JS, Klungel $\mathrm{OH}$, Robinson NJ, Ibáñez L, et al. Potential impact of benzodiazepine use on the rate of hip fractures in five 


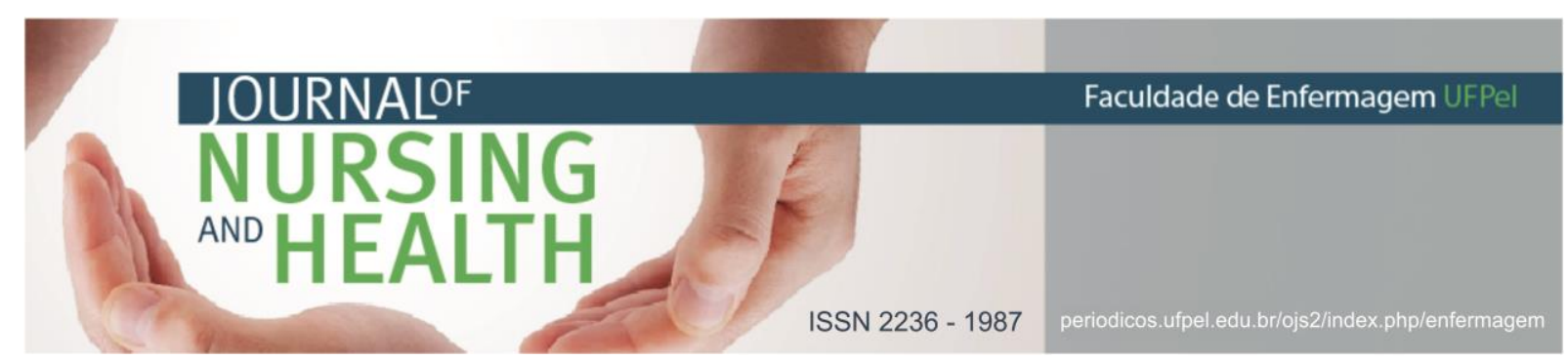

large European countries and the United States. Calcif tissue int [Internet]. 2012[acesso em $2017 \mathrm{fev}$ 10];91(1):24-31. Disponível em: https://www.ncbi.nlm.nih.gov/pmc/a rticles/PMC3382650/

Data da submissão: 2016-09-07 Aceito: 2016-12-05

Publicação: 2017-04-12 